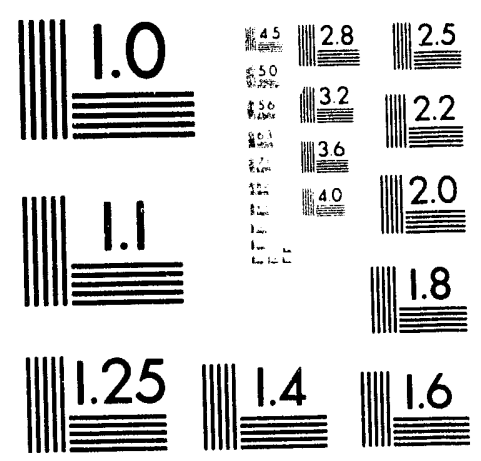



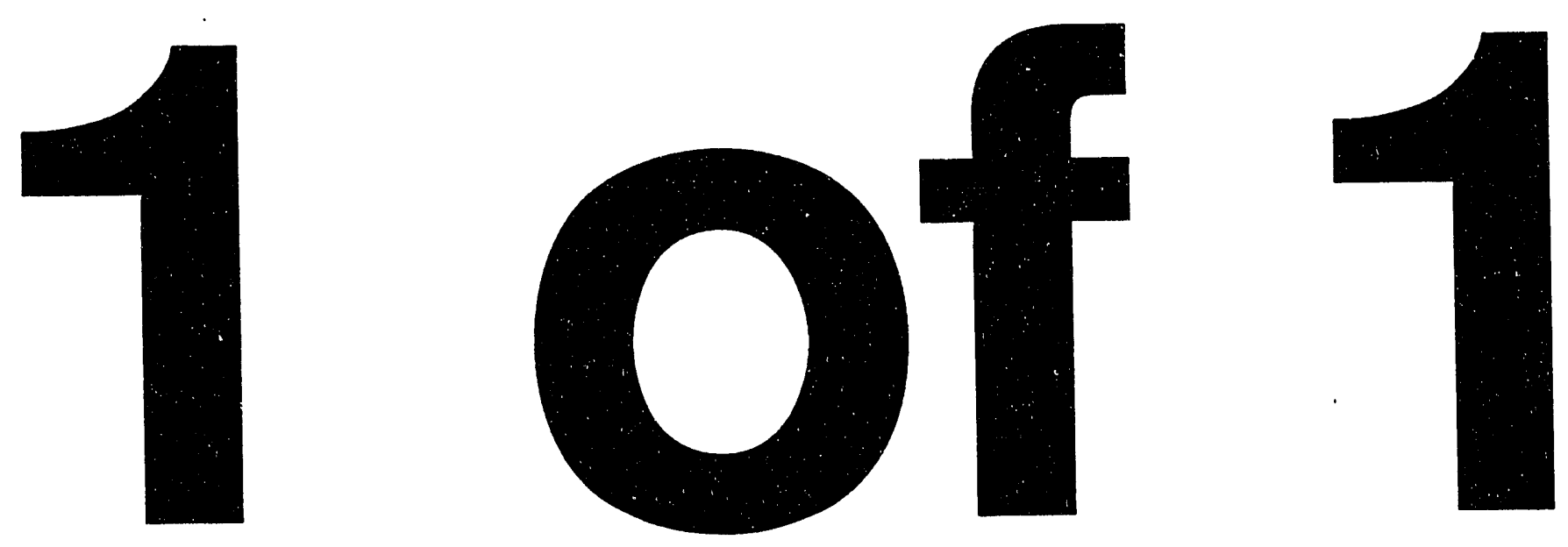
UCRL- JC- 113740

PREPRINT

\section{HIGH ENERGY REAL-TIME IMAGING STUDIES}

J.J. Haskins, K.W. Dolan, D.E. Perkins, D. Rikard, and D.J. Schneberk

This paper was prepared for submittal to the 1993 JANNAF Nondestructive Evaluation Subcommittee Meeting, Livermore, CA

April 26-28, 1993

\section{April 1993}

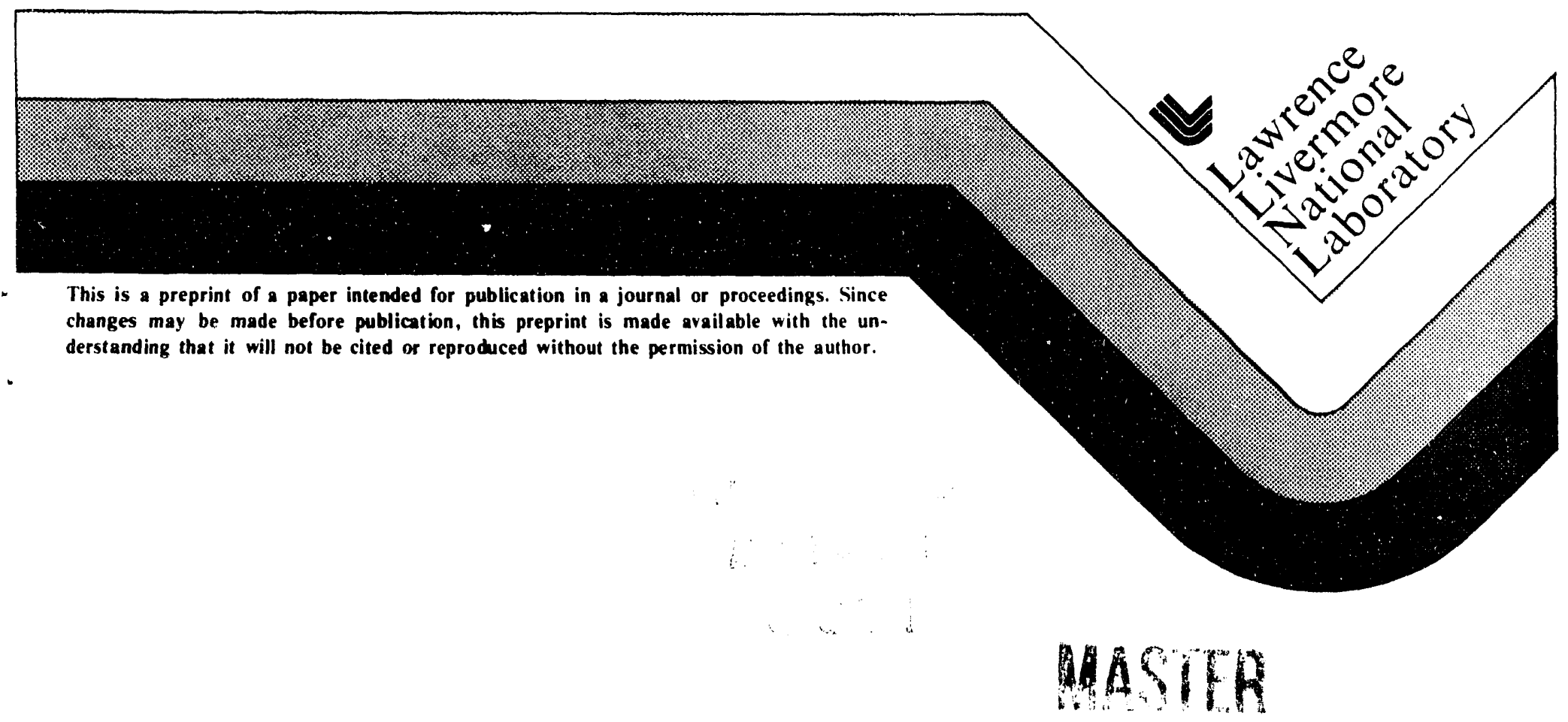




\section{DISCI.AINER}

This document was prepared as an account of work sponsored by an agency of the United States Government. Neither the United States Government nor the University of California nor any of their employees, makes any warranty, express or implied, or assumes any legal liability or responsibility for the accuracy, completeness, or usefulness of any information, apparatus, product, or process disclosed, or represents that its use would not infringe privately owned rights. Reference herein to any specific commercial products, process, or service by trade name, trademark, manufacturer, or otherwise, does not necessarily constitute or imply its endorsement, recommendation, or favoring by the linited States Government or the University of California. The views and opinions of authors expressed herein do not necessarily state or reflect those of the Linited States Covernment thereof, and shall not be used for advertising or product endorsement purposes. 


\title{
HIGH ENERGY REAL-TIME IMAGING STUDIES *
}

\author{
J. J. Haskins, K. W. Dolan, D. E. Perkins, D. Rikard, and D. J. Schneberk \\ Lawrence Livermore National Laboratory \\ P. O. Box 808, Livermore, CA 94550
}

\begin{abstract}
Performance characteristics of high energy real-time radiography (RTR) s\%'stems were optimized by interchanging components and varying optical coupling methods. Phosphor screens, fiber optic scintillation plates, monolithic high density glass scintillation plates, mirror coatings, different cameras and integration times were studied. X-ray sources were 4- and 9-MeV linear accelerators. High density monolithic giass, high resolution and wide dynamic range CCD cameras, and special focusing and fixturing methods have provided significantly improved spatial resolution and contrast for our high energy real-rime imaging. RTR systems with improved performance characteristics and proper translational/rotational staging were adapted for computed tomography applications.
\end{abstract}

\section{INTRODUCTION}

The acquisition and optimization of real-time and near real-time imaging data requires an evaluation of both the inspection goals and the characteristics of each element of the imaging system. In this paper we will identify the important parameters and relationships for transmission imaging using penetrating $(x-$ ray) radiation and present results from our equipment and technique optimization experiments. The image forming process for these inspection techniques is the result of two sub-processes; i) the interaction between the penetrating radiation and the object producing a transmission or virtual image in space, and ii) the process of recording that image with a detection system. An "optimum" inspection technique combines the best configurations of the source of radiation, object position, source-object-detector geometry, and a "matched" detection system that can record all the fidelity contained in the virtual image. By analyzing the imaging process we will discuss different aspects of "optimum" inspection using penetrating radiation.

The ability to interpret transmission images, or to obtain high quality post-process information from image data sets (e.g., image enhancement, or reconstructed computed tomography) directly depends on two properties of the image; the spatial resolution and the contrast sensitivity. For any transmission image, the ability to identify some feature in the image is a combination of the ability to resolve the dimensions of the feature (the spatial resolution), and then to resolve density and thickness variations (the contrast sensitivity). While these two properties are independent measures of image fidelity and provide independent measure of system performance, they are necessarily combined in any particular image [ASTM89].

\section{IMAGE SYSTEM OPTIMIZATION}

\section{DISCUSSION OF OPTIMUZATION}

It would indeed be fortunate if there were an unequivocal understanding of "optimum" inspection performance, but this is rarely the case. While it may be possible to define a configuration that obtains the optimum transmission image (defined in terms of the "best" spatial resolution and "best" contrast sensitivity), this ideal configuration may be impossible to field, or impractical to apply (i.e., the inspection may take too long or require an unachievable radiation source). Our understanding of "optimum" is necessarily based on measures of inspection performance. We have confined our attention to techniques that can be configured with "off-the-shelf" components. In spite of this emphasis, it is recognized that optimization is a matter of discussion where a large number of concerns need to be addressed (throughput, cost, object motion, image enhancement methods, etc.).

Optimization of $\mathrm{x}$-ray image formation is essential for both on-line real-time imaging and subsequent off-line image processing. Much of our experience in obtaining two-dimensional images with real-time and near real-time systems

* Work performed under auspices of the U.S. Department of Energy by the Lawrence Livermore National Laboratory under contract No. W-7405-ENG-48. Approved for public release; distribution unlimited. 
is for the purpose of producing data sets for CT reconstructions. While not discussed here, CT optimization is also an important consideration for our imaging systems (for a discussion of CT systems, see [MART90]). Optimization requires evaluation of the essential elements of the image formation and the image detection process. The formation of the transmission or virtual image in space is determined by both the source characteristics and the object characteristics. Image detection is a separate process requiring the capture of the maximum amount of information contained in the virtual image. The $x$-ray beam plays a major role in generating the transmission image, and every property of the beam is important for RTR inspections. Important properties include beam shape, beam intensity, and beam energy content. The position of the object in the beam is also important, and the beam and the object taken together generate a virtual transmission image in space which has a certain inherent fidelity. Our goal is to identify the important experimental variables for imaging and lay the groundwork for determining different imaging configurations which can be considered optimal.

\section{OPTIMUZATUON PARAMETERS}

Five operational parameters have a direct bearing on optimization. These are source energy, source focal-spot size, source-to-object distance, object-to-detector distance, and image integration time. When slit collimation is introduced for near real-timie scanning with a linear detector array, a number of additional considerations apply. For a two-dimensional (2D) detector, these five parameters represent the dimensions for optimizing real-time performance. Of the five, the energy and object placement offer the widest range of possible choices. Often, the sclection of focal-spot sizes for a given $x$-ray source is limited to one or two choices (e.g., large and small focal spot). For integration time, $33 \mathrm{msec}$ at normal video framing rate provides the standard integration time. This can be varied in some cameras to provide a longer integration times. Frame summing can also be used on-line, or offline in post processing, to provide arbitrarily long integration times. Increasing the integration time results in image formation with a larger number of $x$-rays providing better statistics and better contrast. Long integration times can be incompatible with object motion resulting in image blur, and can become unreasonable long even with stationary objects. The limiting factor for long integration times may also be the dynamic range of the detector. Our method has been to concentrate on assessing the impact on performance of the source energy and object distance parameters optimize on these variables assuming fixed focal-spot size, and then optimize the integration time.

\section{DETECTOR CONSIDERATIONS}

Our investigations have concentrated on $2 \mathrm{D}$ or area-array imaging systems. We have characterized the interaction between the penetrating radiation and the object by assessing the properties of a $2 \mathrm{D}$ detector plane in space. Given our interest in examining the limits of the transmission image in space, it is important that our ? $2 \mathrm{D}$ detector have some provision for changing the pixel size. Since we are interested in high resolution, we necú to have a means for obtaining smaller pixel sizes with our detector package. We have found that detector packages which include solid state cameras which are lens-coupled to a detection medium (e.g. scintillator glass or phosphor screens) are an attractive choice. By using different lenses, it is possible to obtain fields of view as large as 54 inches on-a-side and as small as $3 / 8$ inch.

Independent of camera optics, it is hardly useful to vary the field of view and ohtain smaller pixel dimensions if the inherent spatial resolution of the detection medium cannot hold the fidelity of the image in space. Equally important, if the detection medium does not generate enough brightness for the camera, the acquired images will not reproduce the information in the transmission image. A previous in-house study of phosphor screens (sce Table I) has led us to gadolinium oxysulfide (GUS) loaded screens as the best choice for high energy work. The screen output must also be matched to response of the camera, and for silicon based CCD (charge-coupled device) and SIT (silicon-intensifed tubes) cameras, the GOS screen output matches well (see Figure 1).

Table I. Light intensity for commercial fluorescent screens. *

\begin{tabular}{|l|l|l|l|l|}
\hline \multirow{2}{*}{$\begin{array}{l}\text { Commercial } \\
\text { Screen }\end{array}$} & $\begin{array}{l}\text { Fluorescent } \\
\text { Material }\end{array}$ & $300 \mathrm{kVp}(3.2 \mathrm{~mm} \mathrm{Fe})$ & $4 \mathrm{McV}(16 \mathrm{~mm} \mathrm{Fe})$ & $\mathrm{Co}-60(51 \mathrm{~mm} \mathrm{Fe})$ \\
\hline Trimax 12 & $\mathrm{Gd}_{2} \mathrm{O}_{2} \mathrm{~S}$ & .014 & .001 & .0018 \\
Siemens & $\mathrm{ZnCdS}_{\text {NDT9 }}$ & .0049 & .00073 & .00054 \\
Quanta III & $\mathrm{CaWO}_{4}$ & .0012 & .00011 & .00008 \\
& $\mathrm{LaOBr}$ & .00024 & $.0000) 34$ & .000026 \\
\hline
\end{tabular}


We have used a recently developed and commercially available terbium-activated high-density (HD) clear glass scintillator [PLA91\} that provides inherent spatial resolution of $20-25 \mathrm{line}-\mathrm{pair} / \mathrm{mm}(\mathrm{lp} / \mathrm{mm})$, and enough brightness for CCD cameras. This glass maintains its high performance characteristics into the $\mathrm{MeV}$ range, but with some loss of spatial resolution. We have used the HD glass with low energy ( $160 \mathrm{kVp})$, medium energy ( $320 \mathrm{kVp})$, and high energy ( 4 and $9 \mathrm{MeV}$ ) imaging systems with good results. The HD glass can be fabricated in either a fiberoptic plate or a clear solid monolithic plate. We regularly use the clear solid glass plate at all energies to avoid crosstalk between fibers. Crosstaik between fibers occurs when a single $x$-ray interaction event causes scintillation in adjacent fibers. We have found that a beam divergence as small as 2.4 degrees will introduce crosstalk between fibers even at low energy. Our HD glass plate and fiber-optic plate inventories allow us to cover a field-of-view range from 2 inch by 2 inch, to 12 inch by 12 inch. We have used the HD glass plates, fiber-optic plates and phosphor screens for comparison studies on this project.

\section{DATAACOUISITION SYSTEM}

Digitized data for the post-processing evaluations has been acquired with a PC-AT compatible computer with an Intel 486 processor, 4 Mbytes of random access memory (RAM), standard serial ports, mouse, 3 AT-bus slots, and $320 \mathrm{Mbytes}$ of local disk storage. A dual-width imaging card is installed in the first two slots and is the primary hardware for reading, digitizing, and manipulating the camera signals. The imager card includes up to four $512 \times 512$ single byte memory buffers which can be grouped together in a number of different ways ( 4 each $512 \times 5128$-bit buffers, 2 each $512 \times 512$ 16-bit buffers, 1 each $1024 \times 512$ 16-bit buffer, or a single $2048 \times 512$ 8-bit data store). Full control of gain and offset are provided, as well as 3 separate LUTs (look up tables) for false color imaging. A video rate arithmetic logic unit ( $A L U$ ) is included, and supporting memory and software for image arithmetic, image averaging, summing, a facility for user-defined $3 \times 3$ convolutions, and a number of pre-programmed kernels for edge detection and general image processing. The imager card image-processing library is resident on the local disk in $\mathrm{C}$ compiler code. An ethernet card occupies the last slot in the PC-AT bus.

\section{HIGH ENERGY IMAGING}

A number of imaging system prototypes have been evaluated at 4 and $9 \mathrm{MeV}$. All of the systems are configured with 45 degree mirror to facilitate shielding for the camera and electronics. The various systems are the classified in terms of the scintillator/ detector combination used.

- gadolinium oxysulfide (GOS) particulate screen with SIT camera

- doped fiber optic screen with SIT camera

- HD glass with SIT camera

- HD glass with CCD camera

- doped fiber optic screen with CCD camera

- gadolinium oxysulfide (GOS) particulate screen with intensified-CCD camera

A commercially available CCD camera with $8.4 \times 9.8$ micron pixels in a $768 \times 494$ format is used for most of our ume integrated imaging. It features a high performance interline transfer imager, which reduces dark current, lag, and blooming while improving dynamic range and spectral characteristics. A 1000: l overload capability allows transmission of clear video signals even when abnormally bright signals are present. It can be operated in an RS170 mode or can be set to frame surn, frame integrate, or frame average. The spectral response of the camera peaks at 500 nanometers and has a full spectrum faceplate sensitivity of $0.01 \mathrm{fc}$ at $80 \%$ video (automatic gain control, AGC, on). External access is provicied for manual control of functions such as gamma, electronic shutter, and gain.

The HD scintillating glass is a recent development that provides prompt response $x$-radiographic imaging. The material is a terbium-activated gadolinium-silicate glass with density of $3.75 \mathrm{~g} / \mathrm{cc}$. The dominant emission wavelength is 543 nanometers and the spatial resolution limit is between 20 and $25 \mathrm{lp} / \mathrm{mm}$ below $150 \mathrm{kV}$ and approximately $2 \mathrm{lp} / \mathrm{mm}$ at $9 \mathrm{MeV}$. The glass has a high $\mathrm{x}$-ray absorption efficiency and a high $\mathrm{x}$-ray to light conversion efficiency. Because the material is transparent, thick sections can used at high energies without losing the light in the scintillator matrix. Its light output, decay characteristics, and stability make it especially suitad for video based imaging systems.

The optimum system for non-dynamic events couples a cooled CCD camera with the HD glass. Remote controlled staging is used to focus into the glass rather than on the surface. Frame integration times as long as 8 seconds have been used with no measurable dark current. This combination gives very high quality images at 4 and 
$9 \mathrm{MeV}$ for non-dynamic events. Imaging of dynamic events has been done with phosphor screens and intensifiedCCD cameras. A large area phosphor screen, $51 \times 42$ inches, composed of a nine smaller gadolinium loaded $14 \times$ 17 inch screens, was used with an intensified-CCD camera to obtain 0.05 inch spatial resolution with image definition at 2 rads/min.

\section{CONCLUSIONS}

Significant improvements in high energy real time radiography were obtained with systems using high density monolithic scintillation glass, CCD cameras with variable time integration, and remote focusing. Improved spatial resolution and contrast obtained with these systems allowed optimal use of image data for post-processing, including use of data sets for computed tomography reconstructions. Gadolinium loaded phosphor screens and intensifiedCCD cameras were the basis for large area imaging systems.

\section{REFERENCES}

[ASTM89] "Standard Guide for Radioscopic Real-Time Imaging", Designation: E 100 - 89, August 1989, American Society for Testing and Materials.

[MART90] "Computerized Tomography", H. E. Martz, G. P. Roberson, D. J. Schneberk, and S. G. Azevedo, November-December 1990 issue of Einergy and Technology Review (UCRL-52000-90-11-12), Lawrence Livermore National Laboratory, Livermore, California.

[PLA91] "High Density Glass Scintillaior "ior Real-Time X-Ray Inspection", R. C. Placious, D. Polansky, H. Berger, C. Bueno, S. L. Vosberg, R. A. Betz and D. J. Rogerson, Materials Evaluation, November 1991.

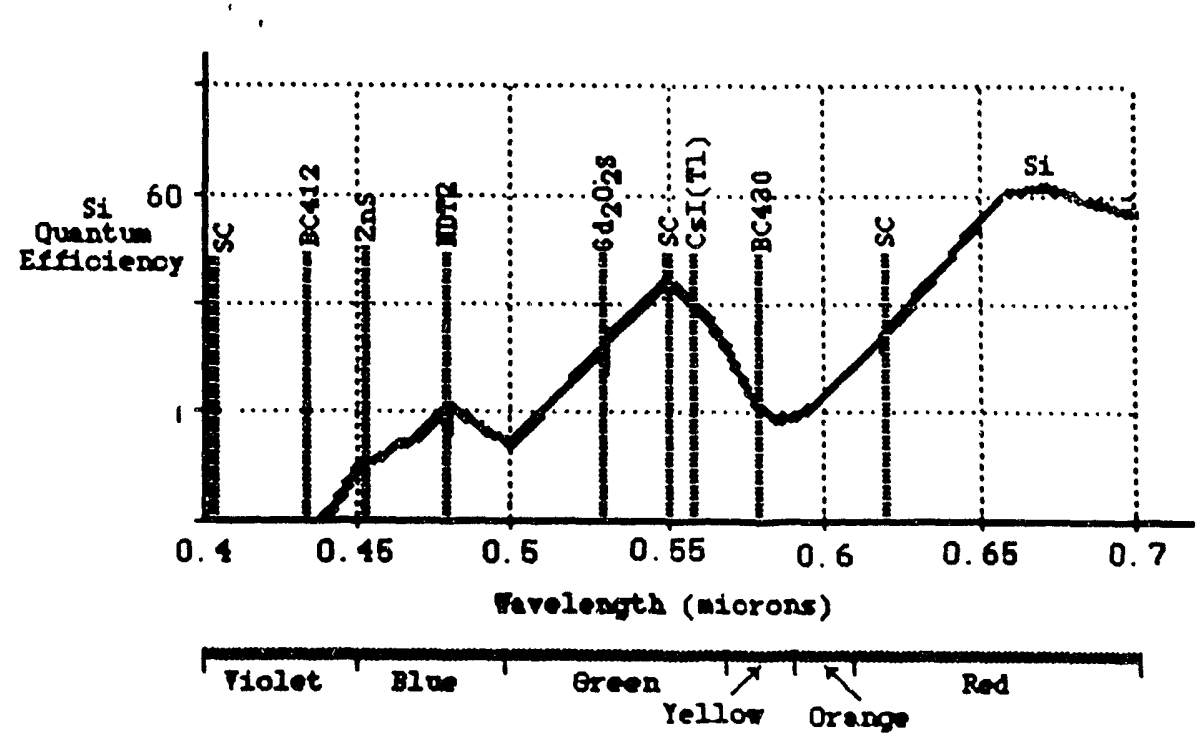

Figure 1. Quantum efficiency of silicon representative of the wavelength response of CCD and SIT cameras with overlays of maximum emission wavelength for differnt tluorescent materials. 

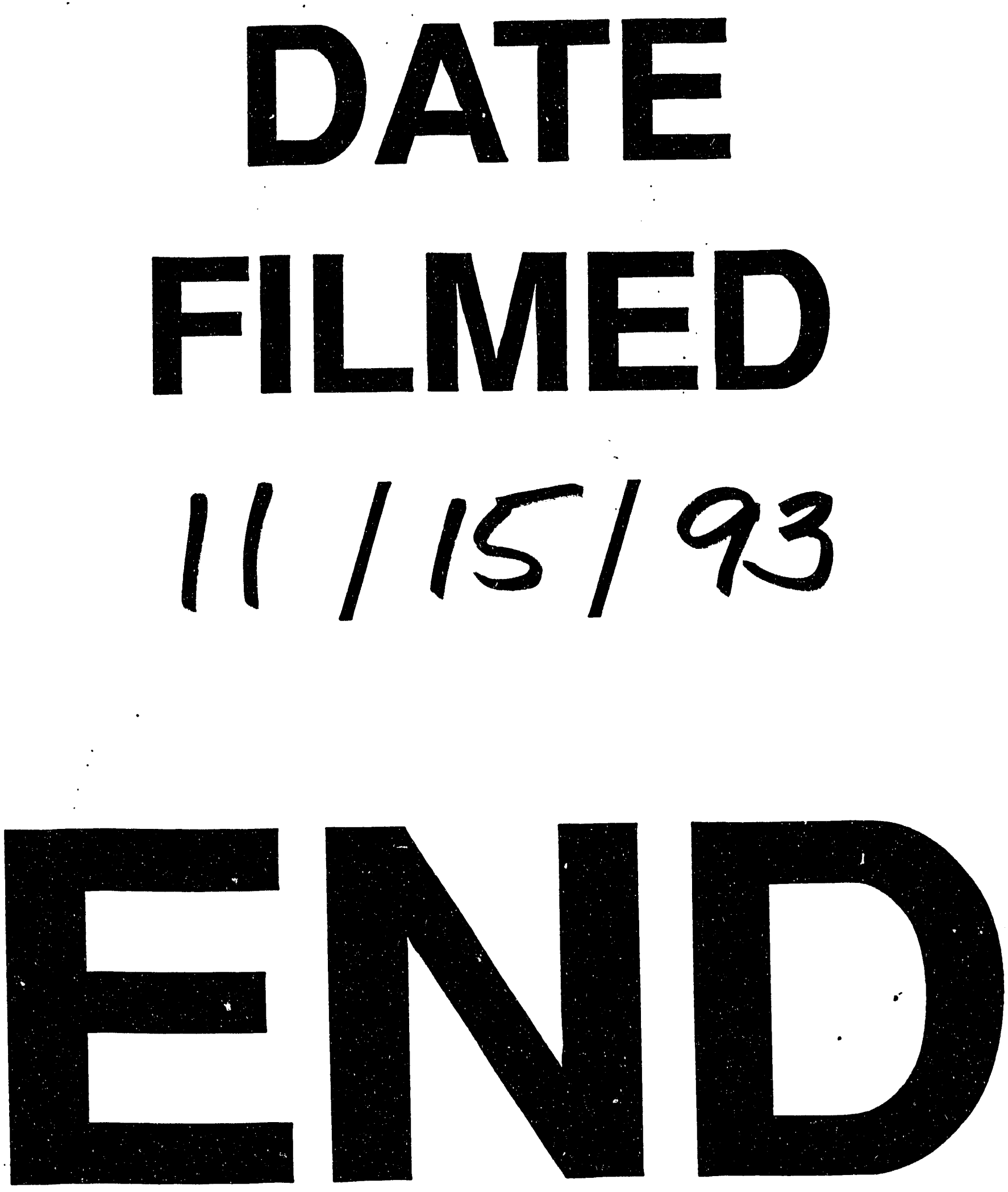
